\title{
Interfacial Self-Assembly to Spatially Organize Graphene Oxide Into Hierarchical and Bioactive Structures
}

\author{
Anna Majkowska ${ }^{1,2,3}$, Carlos Redondo-Gómez ${ }^{2,3}$, Alistair Rice ${ }^{4}$, Mariel Gonzalez $^{3}$, \\ Karla E. Inostroza-Brito ${ }^{3}$, Estelle C. Collin ${ }^{3}$, Jose Carlos Rodriguez-Cabello ${ }^{5}$, \\ Armando E. Del Rio Hernandez ${ }^{4}$, Egle Solito ${ }^{1}$ and Alvaro Mata ${ }^{2,3,6,7,8 *}$
}

${ }^{1}$ Barts and The London School of Medicine and Dentistry, William Harvey Research Institute, Queen Mary University of London, London, United Kingdom, ${ }^{2}$ School of Engineering and Materials Science, Institute of Bioengineering, Queen Mary University of London, London, United Kingdom, ${ }^{3}$ School of Engineering and Materials Science, Queen Mary University of London, London, United Kingdom, ${ }^{4}$ Department of Bioengineering, Imperial College London, London, United Kingdom, ${ }^{5}$ BIOFORGE Group, University of Valladolid, CIBER-BBN, Valladolid, Spain, ${ }^{6}$ School of Pharmacy, University of Nottingham, Nottingham, United Kingdom, ${ }^{7}$ Biodiscovery Institute, University of Nottingham, Nottingham, United Kingdom, ${ }^{8}$ Department of Chemical and Environmental Engineering, University of Nottingham, Nottingham, United Kingdom

OPEN ACCESS

Edited by:

Lorenzo Moroni,

Maastricht University, Netherlands

Reviewed by:

John B. Matson,

Virginia Tech, United States

Marcel Bouvet,

Université de Bourgogne, France

*Correspondence:

Alvaro Mata

a.mata@nottingham.ac.uk

\footnotetext{
Specialty section:

This article was submitted to Biomaterials,

a section of the journal

Frontiers in Materials
}

Received: 27 March 2020

Accepted: 06 May 2020

Published: 16 June 2020

Citation:

Majkowska A, Redondo-Gómez C Rice A, Gonzalez $M$ Inostroza-Brito KE, Collin EC

Rodriguez-Cabello JC, Del Rio Hernandez AE, Solito E and Mata A (2020) Interfacial Self-Assembly to Spatially Organize Graphene Oxide Into Hierarchical and Bioactive Structures. Front. Mater. 7:167. doi: 10.3389/fmats.2020.00167
Multicomponent self-assembly holds great promise for the generation of complex and functional biomaterials with hierarchical microstructure. Here, we describe the use of supramolecular co-assembly between an elastin-like recombinamer (ELR5) and a peptide amphiphile (PA) to organize graphene oxide (GO) flakes into bioactive structures across multiple scales. The process takes advantage of a reaction-diffusion mechanism to enable the incorporation and spatial organization of GO within multiple ELR5/PA layers. Scanning electron microscopy (SEM), transmission electron microscopy (TEM), and ImageJ software were used to demonstrate the hierarchical organization of GO flakes within the ELR5/PA layers and the distribution profiles of GO throughout the ELR5/PA membranes. Furthermore, atomic force microscopy (AFM) revealed improved Young's Moduli of the ELR5/PA/GO membranes compared to the ELR5/PA membranes. Lastly, we investigated biocompatibility of the ELR5/PA/GO membrane via various cell culture methods.

Keywords: graphene oxide, multicomponent self-assembly, peptide amphiphiles, elastin-like recombinamer, hierarchical biomaterials, composite materials

\section{INTRODUCTION}

Self-assembly, the process by which multiple smaller components autonomously interact and organize into larger well-defined structures, plays a crucial role in the way nature creates structure and functionality (Whitesides and Grzybowski, 2002). In an attempt to emulate biological systems, molecular self-assembly is being used to design bioinspired materials with a spectrum of exciting properties such as well-defined nanostructure (Zhang, 2003; Gazit, 2007), precise display of bioactive signals (Webber et al., 2010; Azevedo, 2019), temporal control of signaling (Kumar et al., 2018), and tuneable mechanical properties (Pashuck et al., 2010). Further processing has been used to enhance complexity for example via modulation of the assembly process (Zhang et al., 2010), top-down techniques (Mata et al., 2009; Mendes et al., 2013), or incorporation of multiple bioactive epitopes (Stephanopoulos et al., 2013; Gentile et al., 2017). However, the ability to assemble molecules hierarchically into well-defined macroscopic structures with practical use remains limited. 
Multicomponent self-assembly offers an attractive route to design and engineer materials with molecular precision while increasing complexity and functionality (Draper et al., 2015). For example, the co-assembly of peptide amphiphiles (PAs) bearing either host or guest moieties has been recently used to develop hydrogels with enhanced mechanical properties (Redondo-Gómez et al., 2019). In a different approach, by co-assembling PAs with a megadalton hyaluronic acid, Capito et al. (2008) created stable sacs and membranes with hierarchical nano-to-micro structure. Inspired by this work, we have used PA molecules as self-assembling chaperones to interact with and guide the assembly of different types of molecules such as 1,3:2,4-dibenzylidene-D-sorbitol (DBS) gelators (Okesola et al., 2019), the protein resilin (Okesola et al., 2020a), or hyaluronan/nanoclay composites (Okesola et al., 2020b), generating hydrogels with tuneable structure and mechanical properties. Furthermore, taking advantage of hydrodynamic forces generated during additive manufacturing, Hedegaard et al. (2018) developed biocompatible hydrogel constructs with well-defined ordered or randomly oriented nanofibers, surface microtopographies, distinct microgeometries, and macroscopic assemblies.

Multicomponent self-assembly offers the possibility to not only take advantage of the properties of the individual components but also emergent assembling phenomena and synergistic properties (Okesola and Mata, 2018). In this context, Inostroza-Brito et al. (2015) demonstrated how PAs can affect the conformation of elastin-like recombinamers (ELRs) to consequently generate a diffusion-reaction assembly process. This mechanism enables the formation of a hierarchical multilayered ELR5/PA membrane with the capacity to access nonequilibrium and a series of dynamic properties. While the resulting material is fragile (Inostroza-Brito et al., 2017), the study demonstrates the possibility to guide the assembly of complex components, such as proteins, beyond the nanoscale in a controllable and autonomous manner.

Graphene oxide (GO) is a single layer two-dimensional nanomaterial with a wide range of properties such as high surface area, mechanical strength, thermal conductivity, biocompatibility, and ease of functionalization (Zhu et al., 2010; Yang et al., 2013). Graphene-based materials have enormous potential in the biomedical field in applications ranging from biosensors (Justino et al., 2017) and biological imaging (Lin et al., 2016) to drug and gene delivery (Liu et al., 2013) and biomaterials (Shin et al., 2016). A variety of composite biomaterials incorporating a biomacromolecule and GO have been generated in the form of electrospun mats (Azarniya et al., 2016), hydrogels (Kang et al., 2015; Zhou et al., 2017), films (Han et al., 2011), or other 3D structures (Rajan Unnithan et al., 2017). For instance, nanocomposites of GO and chitosan have been prepared resulting in improved mechanical properties (Han et al., 2011; Li et al., 2013), resistance against enzymatic degradation (Shao et al., 2013), enhanced cellular (Depan et al., 2014; Dinescu et al., 2014), and antibacterial (Mazaheri et al., 2014) activity. Other GO-protein composite materials based on GO and gelatin or collagen have led to materials with improved mechanical properties (Wan et al., 2011; Jalaja et al., 2016) and bioactivity (Kang et al., 2015; Lee et al., 2016; Zhou et al., 2017). While these examples elucidate both the interest and progress of incorporating GO within biomaterials, the capacity to organize GO flakes hierarchically remains an unmet challenge.

Here, we report on the use of the ELR5/PA co-assembling system to enable localization and organization of GO flakes into hierarchical and functional structures. We demonstrate how the diffusion-reaction mechanism of formation can be used to guide the assembly of GO flakes between the ELR5/PA layers and generate complex geometries where GO is organized at multiple length scales. Furthermore, we reason that the assembly of GO in this manner will lead to enhanced mechanical properties of the ELR5/PA system. We describe the underlying mechanism of self-assembly, the structure of the composite material, and the biocompatibility of the resulting ELR5/PA/GO biomaterial through extensive in vitro cell studies.

\section{RESULTS AND DISCUSSION}

\section{Rationale}

The approach is based on the ELR5/PA co-assembling system (Inostroza-Brito et al., 2015, 2017) and takes advantage of its reaction-diffusion mechanism to recruit, localize, and organize GO flakes at multiple length scales (Figure 1). We used $0.04 \%(\mathrm{v} / \mathrm{v})$ GO (Wick et al., 2014) ( $\mathrm{pH}=2$ ) with monolayer content $>95 \%$ and oxygen content $>36 \%$ given its water dispersability and low cost (Figure 1E). We used a cationic PAK3 $\left(\mathrm{C}_{15} \mathrm{H}_{31} \mathrm{CONH}-\mathrm{VVVAAAKKK}-\mathrm{CONH}_{2}\right)$ to co-assemble with the oppositely charged ELR5 (MESLLP-[(VPGVG VPGVG VPGEG VPGVG VPGVG) 10 -(VGIPG) 60$]_{2}$ - $\left[(\mathrm{VPGIG})_{10}\right.$ AVTGRGDSPASS(VPGIG) $\left.)_{10}\right]_{2}-\mathrm{V}$ ) molecule to evaluate the capacity of the assembling process to recruit and organize GO flakes within the distinctive ELR5/PA multilayers (Figures 1A-C). Furthermore, given our previous findings that distinct PA molecules can generate different ELR5/PA membrane structures (Inostroza-Brito et al., 2015), PAs with different charge densities including PAK2 $\left(\mathrm{C}_{15} \mathrm{H}_{31} \mathrm{CONH}-\mathrm{VVVAAAKK}\right.$ $\left.\mathrm{CONH}_{2}\right)$ and PAK4 $\left(\mathrm{C}_{15} \mathrm{H}_{31} \mathrm{CONH}-\mathrm{VVVAAAKKKK}-\mathrm{CONH}_{2}\right)$ were also used to co-assemble with ELR5 (Figure 1D). In addition, the resulting materials were characterized according to their mechanical properties and used as cell culture substrates to investigate their effect on cell adhesion, proliferation, metabolic activity, and morphology.

\section{Synthesis of Individual Components}

PA molecules were synthesized following standard solid-phase peptide synthesis methods as previously reported (Mata et al., 2012). PA purity and structure were characterized by reverse phase HPLC and electrospray ionization mass spectrometry (Figure S1). ELR5 molecules were obtained from Technical Proteins Nanobiotechnology S. L., Spain and GO aqueous dispersions were obtained from Sigma Aldrich, UK.

\section{Characterization of Interactions Between Components}

The ELR5/PA system relies on electrostatic, hydrophobic, and $\mathrm{H}$ bond interactions (Inostroza-Brito et al., 2015). Consequently, we 


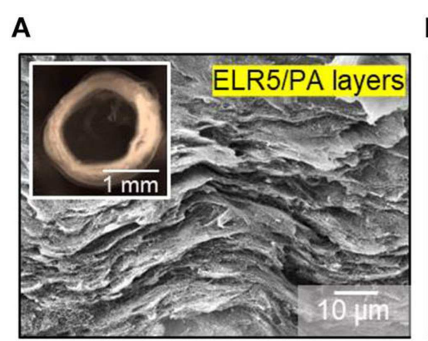

B

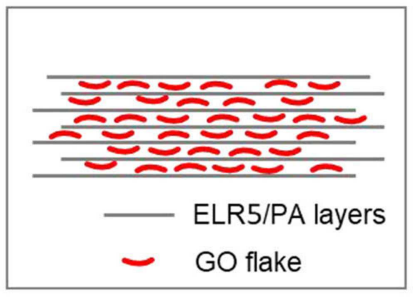

C

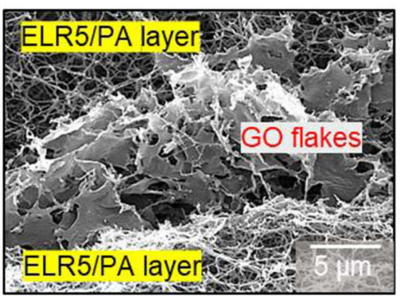

D

\begin{tabular}{|c|c|c|}
\hline MOLECULE & SEQUENCE & $M_{w}(k D a)$ \\
\hline PAK2 & $\mathrm{C}_{15} \mathrm{H}_{31} \mathrm{CONH}$-VVVAAAKK-CONH ${ }_{2}$ & 1.022 \\
\hline PAK3 & $\mathrm{C}_{15} \mathrm{H}_{31} \mathrm{CONH}$-VVVAAAKKK-CONH ${ }_{2}$ & 1.150 \\
\hline PAK4 & $\mathrm{C}_{15} \mathrm{H}_{31} \mathrm{CONH}$-VVVAAAKKKK-CONH ${ }_{2}$ & 1.279 \\
\hline ELR5 & 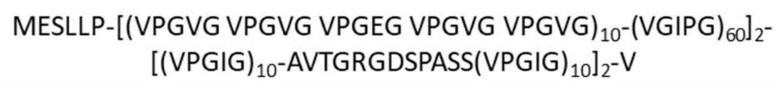 & 112.300 \\
\hline
\end{tabular}

\begin{tabular}{l} 
E GRAPHENE OXIDE \\
\hline $\mathrm{pH} 2$ \\
Lateral size: $7 \pm 2 \mu \mathrm{m}$ \\
Monolayer content: $\geq 95 \%$ \\
Zeta potential: $-30.58 \mathrm{mV}$ \\
Oxygen content: $\geq 36 \%$
\end{tabular}
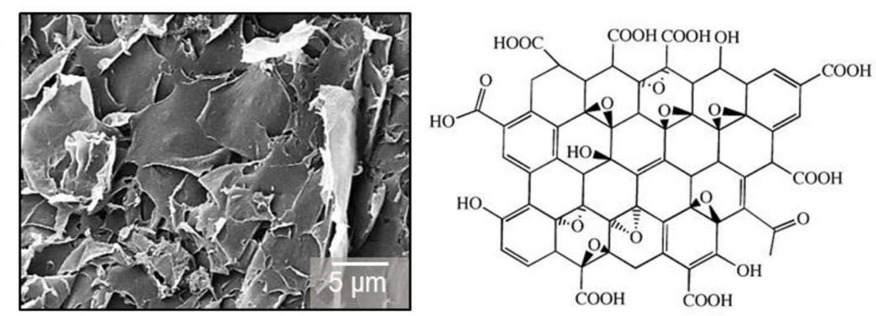

FIGURE 1 | Schematic representation and representative images of tubes and tube wall cross-sections of the ELR5/PA/GO system. (A) SEM micrograph of an ELR5/PAK3 cross-section with its characteristic multi-layered architecture. Inset: Bright field image of a top view of an ELR5/PA tube. (B) Schematic of the ELR5/PA system cross-section with black horizontal lines representing ELR5/PA layers embedded with GO flakes (red). (C) SEM micrograph of the cross-section of an ELR5/PA/GO membrane visualizing the localization of GO flakes between ELR5/PA layers. (D) Molecular structure and weight of PAs and ELR5 used in this study. (E) Molecular information and structure of GO used in this work.

first used circular dichroism (CD) to investigate the secondary structure in aqueous solution of both PAs and ELR5 with and without GO. CD revealed that PAK3 exists in a random coil conformation when dissolved in MilliQ ${ }^{\mathrm{TM}}$ water at $\mathrm{pH} 4.5$ and room temperature (RT), and does not undergo conformational change when mixed with GO under the same conditions (Figure 2A). However, a slight red-shift at the $195 \mathrm{~nm}$ minimum was observed, which might indicate an interaction between PAK3 and GO as previously reported (Pashuck et al., 2010). $\mathrm{CD}$ on ELR5 samples dissolved in MilliQ ${ }^{\mathrm{TM}}$ water at $\mathrm{pH} 5$ and RT exhibited both random coil and $\beta$-sheet conformations (Figure 2A) that did not change upon addition of GO. However, lower intensity of the negative $195 \mathrm{~nm}$ signal might indicate that GO reduces the content of random coil structures in ELR5 while increasing the content of type I $\beta$-turns (Perczel and Fasman, 1992).

We further investigated the ELR5/PA/GO interactions by conducting zeta potential and dynamic light scattering (DLS) measurements. Zeta potential of PAK3 decreased after addition of GO (Figure 2B). This is likely a consequence of a drop in the
PAK3's surface charge as a result of its electrostatic interactions with carboxyl, hydroxyl, or carbonyl groups present in GO (negatively charged), partially screening the positive charges of PAK3. On the other hand, the zeta potential of ELR5 molecules increased slightly after addition of GO, suggesting absence of electrostatic interactions between these components, which is in agreement with the CD results (Figure 2A). DLS measurements revealed a dramatic decrease in GO size after mixing with both PAK3 (Figure 2C) and ELR5, which suggests disruption of GO aggregates, known to form in aqueous solutions (Tang et al., 2015), due to electrostatic interactions. It is important to mention that DLS is a well-suited technique for estimating the size of spherical particles. However, this technique has been also used to provide relative changes in size of non-spherical components including GO (Stankovich et al., 2006), PAs (Raymond and Nilsson, 2018), and ELRs (Navon and Bitton, 2016).

Hydrophobic interactions also play a key role in the ELR5/PA system above the ELR5's transition temperature (Tt) (InostrozaBrito et al., 2015). To investigate whether addition of GO into the ELR5/PA system influences hydrophobic interactions, a turbidity 

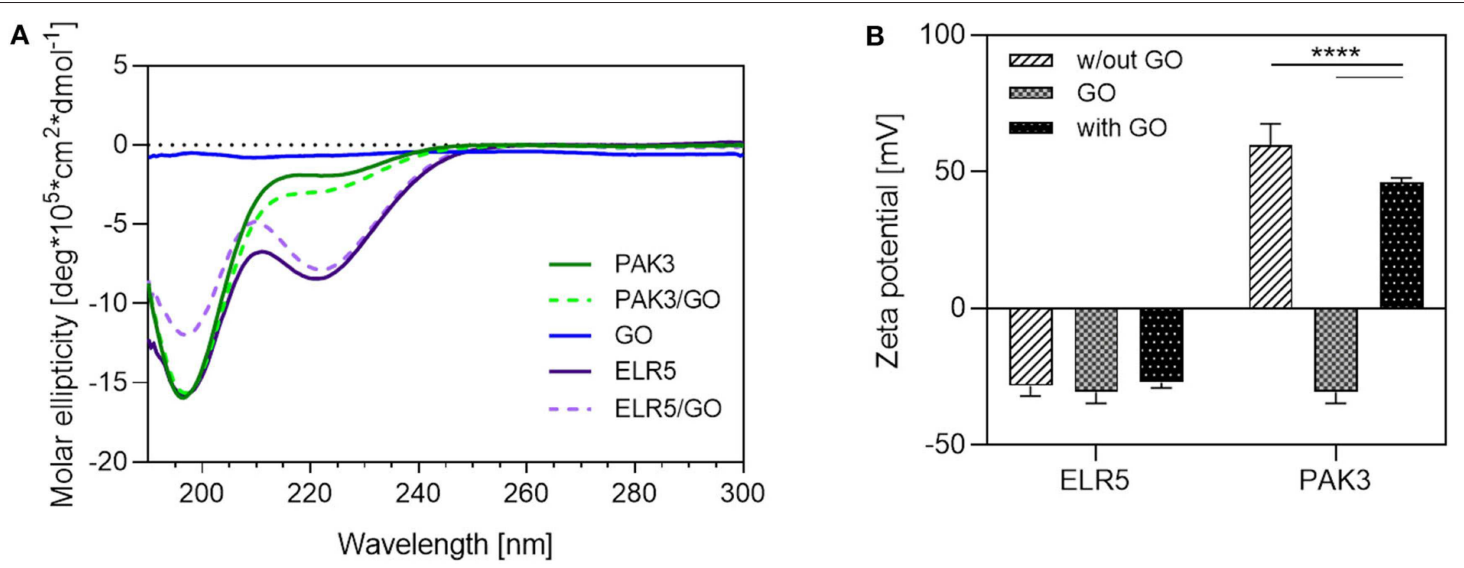

C

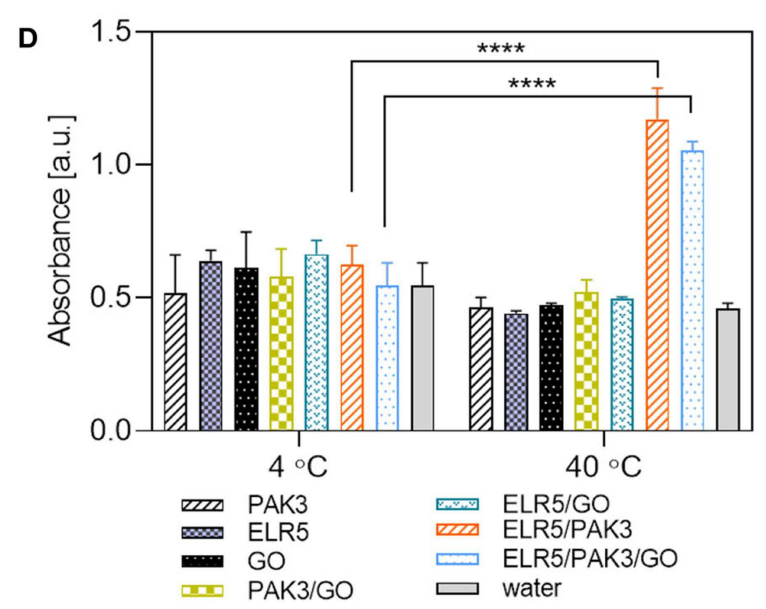

FIGURE 2 | Analysis of interactions between PAK3, ELR5, and GO. (A) CD spectra of PAK3, ELR5, and their mixtures with GO in aqueous solutions at room temperature. (B) Zeta potential measurements of aqueous solutions of PAK3, ELR5, and their mixtures with GO. (C) DLS measurements of aqueous solutions of PAK3, ELR5, and their mixtures with GO (D) Turbidity of aqueous solutions of PAK3, ELR5, and their mixtures with GO at 4 and $40^{\circ} \mathrm{C}$ measured at 300 nm. Error bars represent $\pm S D$ where ${ }^{\star * * *} p<0.0001$. The experiments were performed in triplicates.

assay was performed. The results confirmed that significant levels of aggregation between ELR5, PAK3, and GO are only present when the temperature is above the $\mathrm{Tt}$ (19C) of ELR5 (Figure 2D), which indicates that presence of GO in the solution does not influence the hydrophobic interactions between PAK3 and ELR5, necessary for membrane formation (Inostroza-Brito et al., 2015). Analysis of interactions between ELR5, GO and both PAK2 and PAK2-K4 is presented in Figures $\mathbf{S} 2$ and $\mathbf{S 3}$.

These results indicate that molecular interaction between GO and both molecular building blocks (PAK3 and ELR5) relies mostly on electrostatic forces. However, both components retain most of their secondary structures in presence of GO and, consequently, hydrophobic interactions from ELR5 seem to also play a key role above the ELR5 transition temperature.

\section{The ELR5/PA/GO Membrane-Micro and Macroscopic Properties}

Upon ELR5/PAK3 co-assembly, a diffusion barrier is formed across which the PAK3 diffuses (Figure 3A) (Inostroza-Brito et al., 2015). Electron microscopy was used to investigate the assembly of GO within the ELR5/PAK3 membrane when added into the system. We first mixed GO with the PAK3 solution and subsequently inoculated it into the ELR5 solution. Scanning electron microscopy (SEM) revealed poor incorporation of GO (Figure 3B), likely as a result of the inability of the relatively large GO flakes to diffuse through the ELR5/PAK3 diffusion barrier. We then reasoned that combining GO with ELR5 solution might surpass this obstacle. We prepared an ELR5/GO solution followed by inoculation of PAK3 into the mixture. In this case, we observed much higher incorporation of GO in the final membrane (Figure 3C). Given these findings, we then mixed GO with both ELR5 and PAK3 solutions, followed by inoculation of PAK3/GO in the ELR5/GO solution. In this setup, membranes qualitatively exhibited the highest incorporation of GO (Figure 3D) while maintaining their multi-layered structure and capacity to adhere to interfaces and open controllably, transforming sacs into tubular structures (Figures 4C-E). Transmission electron microscopy (TEM) revealed presence of GO flakes (visible as black lines) throughout the thickness of the ELR5/PAK3/GO membrane, positioned within and parallel to the membrane layers (Figure 4B). SEM performed on the 


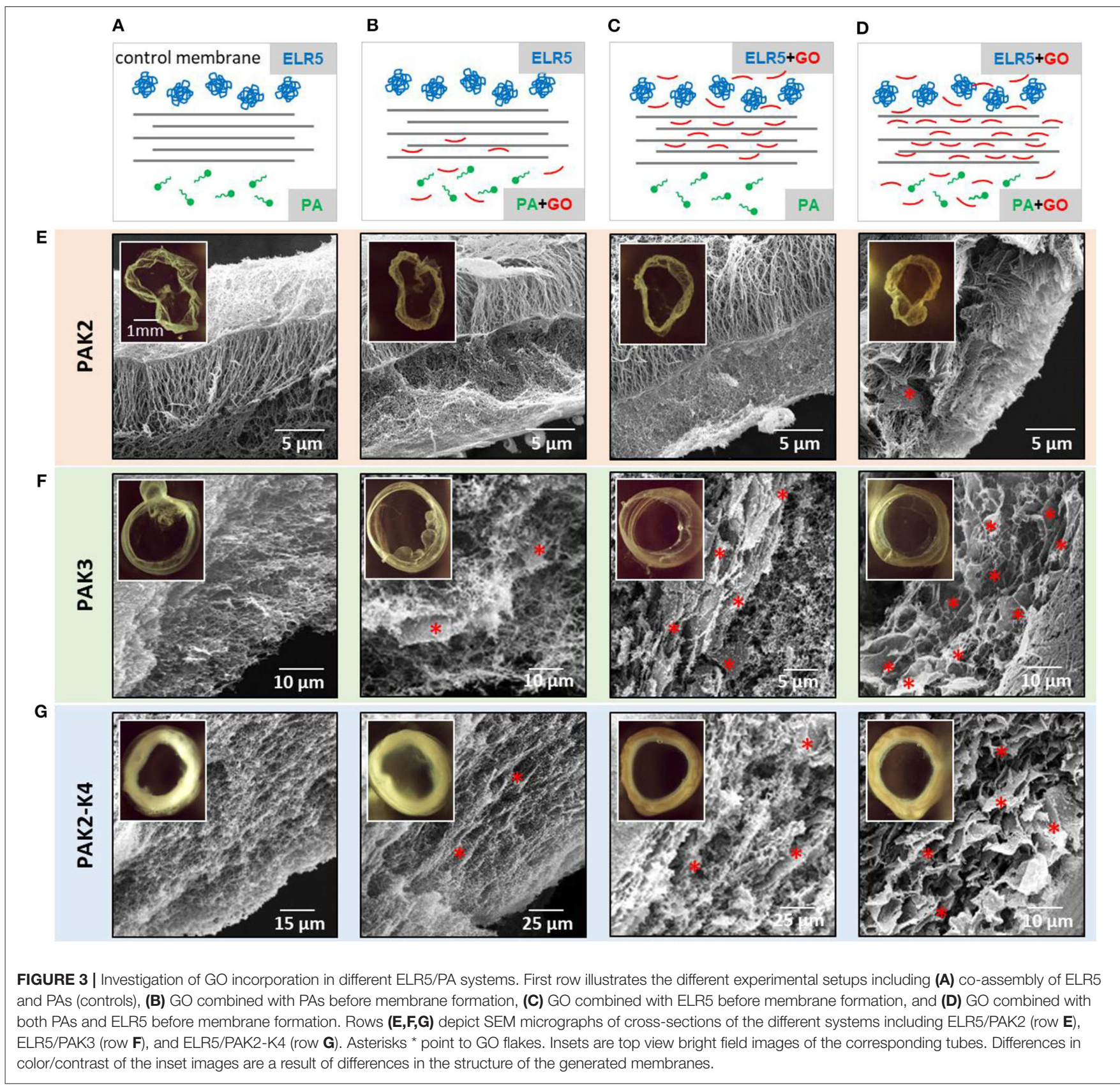

membranes confirmed the multi-layered microarchitecture with embedded GO flakes (Figure 3F-row) seen on TEM. These results indicate that $\mathrm{GO}$ can be successfully incorporated within the ELR5/PAK3 system by taking advantage of the diffusionreaction mechanism of assembly to recruit, localize, and organize GO flakes within the multi-layered architecture and as part of a more complex geometrical structure.

\section{Incorporation Studies}

To investigate the possibility to use supramolecular selfassembling processes to guide the organization of GO more broadly, we repeated the experiments using variations of PAK3,
PAK2 (C15H31-VVVAAAKK-CONH2) and PAK4 (C15H31VVVAAAKKKK-CONH2) (Figure 1D). We have previously demonstrated that these PA molecules with a different number of lysine residues were also able to form stable ELR5/PA membranes, but exhibiting different cross-sectional architectures (Inostroza-Brito et al., 2015). Membranes formed using PAK4 exhibited a multi-layered architecture with increased thickness and a looser structure compared to ELR5/PAK3 membranes (Inostroza-Brito et al., 2015) while those formed using PAK2 exhibited a different three-level structure with orthogonal fibers (Figure 3E-row) (Inostroza-Brito et al., 2015). To study the interaction between GO and the ELR5/PAK2-K4 system, we 

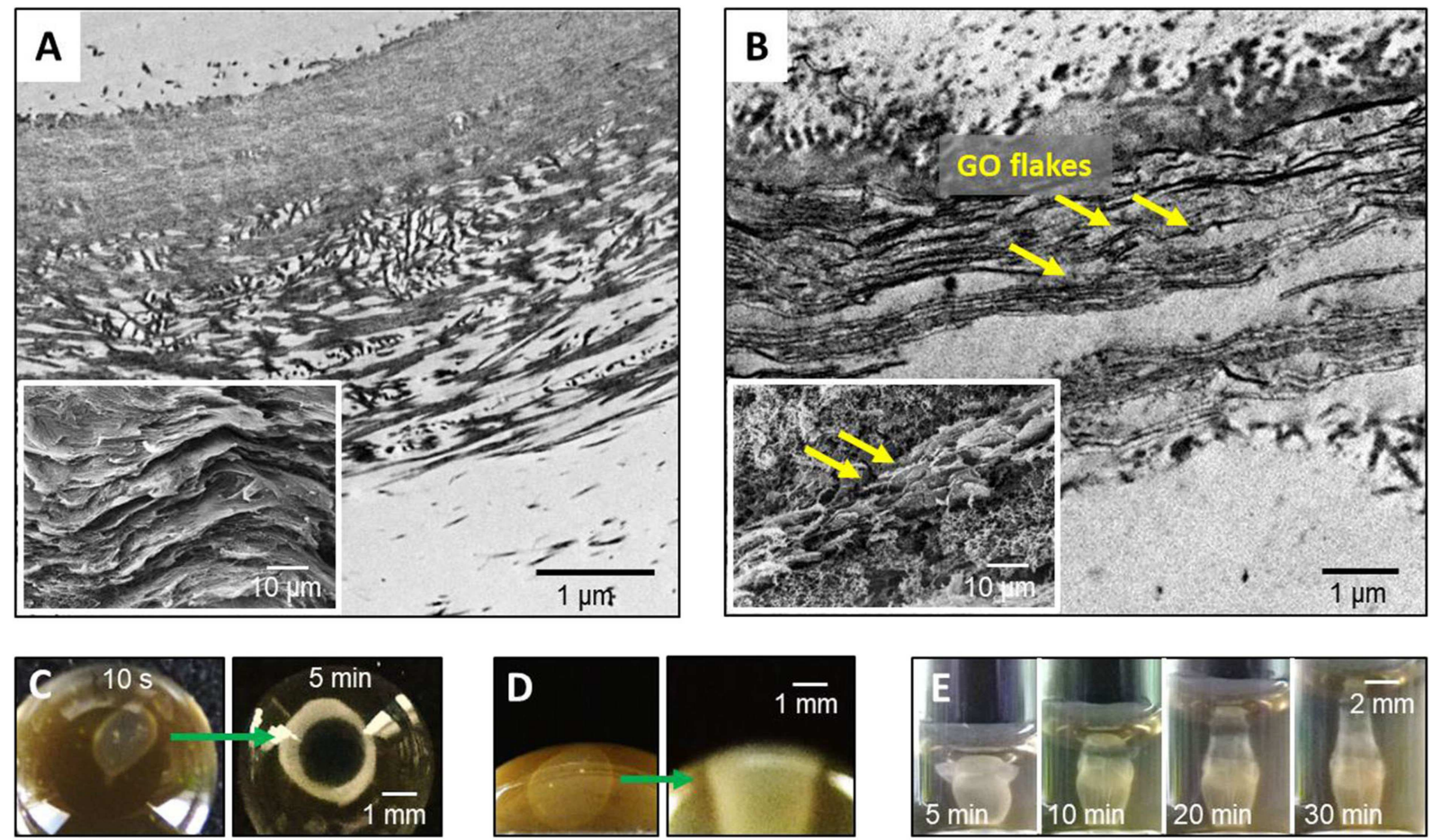

FIGURE 4 | Investigation of GO incorporation and ELR5/PAK3/GO membrane properties. (A) TEM micrograph of a cross-section of an ELR5/PAK3 membrane (Inset is a representative SEM micrograph). (B) TEM micrograph of a cross-section of an ELR5/PAK3/GO membrane with GO flakes (yellow arrows) (Inset is a representative SEM micrograph). (C-E) Demonstration of the dynamic properties of the ELR5/PAK3/GO system including spontaneous opening after a drop of PAK3/GO solution is inoculated into an ELR5/GO solution (C-E) capacity for longitudinal growth upon increasing the ELR5/GO volume.

also attempted to generate ELR5/PA membranes combining both PAK2 and PAK4 (1:1 mixture). We hypothesized that the different diffusion mechanisms arising from the co-assembly of these different components would guide and organize GO flakes differently. To investigate this hypothesis, experiments were conducted using ELR5/PAK2 and ELR5/PAK2-K4 combinations (Figures 3E,G-rows). As before, GO was mixed with (i) PAs alone, (ii) ELR5 alone, and (iii) both components (Figures 3B-D, columns). ELR5/PAK2/GO and ELR5/PAK2-K4/GO membranes were stable and robust (Figures 3E,G insets) and exhibited darker color suggesting GO incorporation in these conditions, which was assessed by observations under an optical microscope. SEM investigations on membranes revealed GO flakes were incorporated and organized within and throughout the multilayered architecture of the ELR5/PAK2-K4/GO membranes (Figure 3G-row). As with the ELR5/PAK3/GO system, highest incorporation of GO was achieved when the $\mathrm{GO}$ was mixed with both PA and ELR5 solutions (Figures 3D,F). In contrast, ELR5/PAK2/GO membranes exhibited large quantities of GO on the outer and inner side of the membrane but only marginal incorporation within the membrane.

These results suggest that GO can be incorporated within the ELR5/PA system independently of the PA molecule used. However, the level of incorporation of GO within the membrane depends on the supramolecular mechanism of assembly, with both ELR5/PAK3/GO and ELR5/PAK2-K4/GO presenting more $\mathrm{GO}$ in the multilayers than ELR5/PAK2/GO membranes, which present a different mechanism of co-assembly (Inostroza-Brito et al., 2015). Initial formation of a strong diffussion barrier prevents large flakes of GO from diffusing through the barrier, forming membranes with less GO flakes. Addition of GO flakes in the ELR5 solution helps to overcome this obstacle, forming membranes with higher content of GO. Highest incorporation, however, can be achieved only when GO is combined with both PA and ELR5 solutions, particularly in the case of the ELR5/PAK3 and ELR5/PAK2-K4 systems.

\section{Membrane Thickness}

To study the effect of introducing GO into the ELR5/PA system, possible changes in membrane thickness were investigated by SEM (Figure 5B). SEM revealed that the thickness of ELR5/PAK3/GO membranes increased when GO was added in either PA or ELR5 solutions prior to assembly, forming the thickest membrane when GO was mixed with both ELR5 and PAK3 (Figure 5D). Similar results were observed in ELR5/PAK2-K4/GO membranes (Figure 5D). In contrast, the thickness of ELR5/PAK2/GO membranes gradually decreased with addition of GO to either PAK2, ELR5, or both PAK2 and ELR5 solutions. This result suggests that the supramolecular mechanism of assembly of ELR5/PAK2 membranes, which differs from ELR5/PAK3 and ELR5/PAK4 systems (InostrozaBrito et al., 2015), prevents incorporation of GO. These experiments are in alignment with the SEM observations (Figure 3) and confirm that, while supramolecular processes 

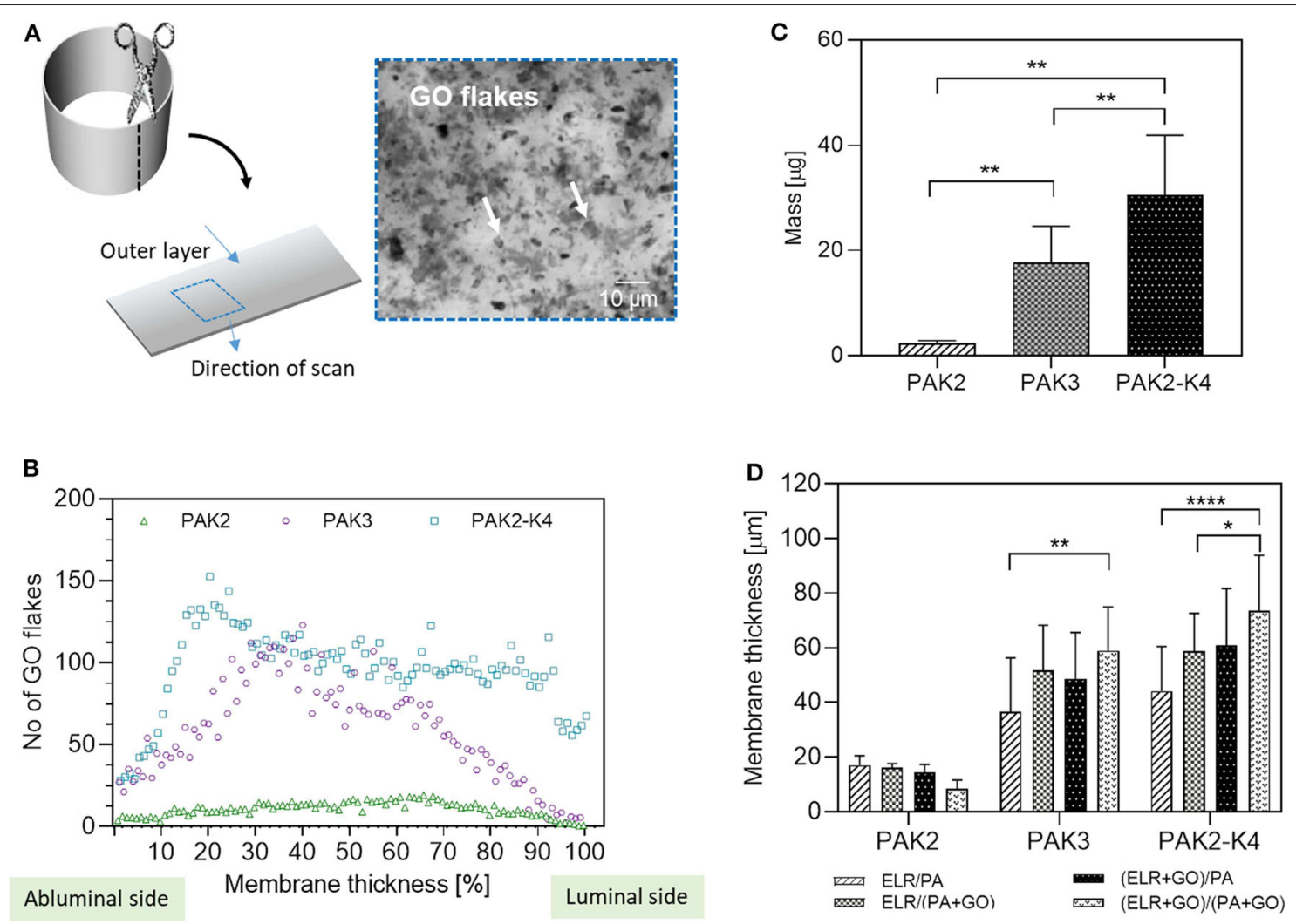

FIGURE 5 | GO content and distribution in the membranes. (A) Schematic of the experimental setup for measuring GO distribution. (B) GO distribution profile throughout the thickness of the ELR5/PAs/GO systems. Number of GO flakes present in each layer of a z-stack image was quantified and presented as number of GO flakes vs. layer number which was normalized as \% of the thickness of the membrane. (C) GO content per membrane as measured by UV-Vis. (D) Thickness of the membranes as measured by SEM. Error bars represent \pm SD where ${ }^{\star \star \star \star} p<0.0001,{ }^{\star \star} p<0.01$, and ${ }^{\star} p<0.5$. The experiments were performed in triplicates.

may be able to organize GO flakes at multiple scales (ELR5/PAK3 and ELR5/PAK2-K4 systems), the size of GO flakes may limit its incorporation in some co-assembling systems (ELR5/PAK2).

\section{GO Content}

To further characterize GO incorporation, the amount of GO within each of the ELR5/PA systems was quantified. After co-assembly, membranes were dissolved in 1,1,1,3,3,3hexafluoro-2-propanol (HFIP) and GO absorbance was monitored spectrophotometrically in the UV-Vis region. The technique enabled quantification of mass of GO per membrane (Figure 5C) and the results revealed the highest incorporation of GO occurred in ELR5/PAK2-K4 membranes, followed by ELR5/PAK3 membranes, and, as expected, ELR5/PAK2 membranes with the lowest incorporation of GO. These results correlate with the previously discussed SEM observations (Figure 3) and membrane thickness experiments (Figure 5B).

\section{Distribution of GO}

SEM and TEM demonstrated that GO flakes are incorporated and organized within the ELR5/PAK3/GO and ELR5/PAK2-K4/GO membranes. To further characterize the level of incorporation throughout the thickness of the membrane, 3D z-stacked bright field images of the membrane were generated (Figure 5A). Using ImageJ software (Schneider et al., 2012), we quantified the number of GO flakes in each layer of the different membranes by generating z-stack images and producing distribution profiles depicting number of GO flakes vs. layer number, which was normalized as $\%$ of the thickness of the membrane (Figure 5B). These profiles revealed that in ELR5/PAK3/GO membranes, GO is distributed throughout the thickness of the membrane with higher amounts present in the middle. In addition, ELR5/PAK2K4/GO membranes exhibited similar level of GO incorporation but the flakes were more evenly distributed throughout the membrane (Figure 5B). Interestingly, ELR5/PAK2/GO membranes exhibited uniform distribution throughout the membrane but with much lower levels of incorporation (Figure 5B). These results confirm that GO flakes were in fact distributed throughout the membranes of all the systems studied but with different levels of incorporation. Highest incorporation and distribution was observed in ELR5/PAK2K4/GO and ELR5/PAK3/GO membranes, on which we focused to assess applicability. 


\section{Mechanical Properties of the ELR5/PA/GO Systems}

Given the unique mechanical properties of GO (Zhu et al., 2010) as well as its incorporation and organization within the ELR5/PA systems, we hypothesized that the mechanical properties of the resulting ELR5/PA/GO membranes would improve compared to the control ELR5/PA membranes. Atomic force microscopy (AFM) measurements were conducted on both luminal (inner) and abluminal (outer) sides of ELR5/PAK3/GO and ELR5/PAK2-K4/GO membranes assembled with GO added to both PA and ELR5 solutions (Figure 6). The results revealed significant increase in Young's Moduli of the ELR5/PAK2-K4/GO membrane on both luminal and abluminal sides compared to ELR5/PAK2-K4 membranes (Figure 6B). This increase was also evident in ELR5/PAK3/GO membranes compared to ELR5/PAK3 ones (Figure 6A). These results suggest that stiffness of the ELR5/PA membrane increases after incorporation of GO on both sides of the membrane, which correlates with the SEM observations (Figure 3), membrane thickness measurements (Figure 5D), and GO distribution and incorporation (Figure 5B) within the membrane's microstructure when GO is added to both ELR5 and PAs solutions.

\section{Cell Studies}

\section{Cell Adhesion}

The potential applicability of the ELR5/PA/GO materials was investigated by assessing the suitability of the membrane to be used in tissue engineering applications. Mouse adipose derived stem cells (mADSCs) were cultured on both sides of the membranes that presented higher incorporation of GO including ELR5/PAK3 (control), ELR5/PAK3/GO, ELR5/PAK2-K4, and ELR5/PAK2-K4/GO. Preliminary biocompatibility was assessed by quantifying cell adhesion, viability, and proliferation.

mADSCs were seeded on both ELR5/PAK3 and ELR5/PAK2$\mathrm{K} 4$ membranes (with and without GO) in serum-free media, incubated for $4 \mathrm{~h}$, rinsed to remove non-adherent cells, cultured for an additional $4 \mathrm{~h}$ in full media (DMEM, 20\% FBS), and then dyed with the blue dsDNA stain 4'-6-diamino-2phenylindole (DAPI). Fluorescent microscopy revealed higher numbers of cells growing on ELR5/PAK3 and ELR5/PAK3/GO membranes than on ELR5/PAK2-K4 and ELR5/PAK2-K4/GO membranes (Figure 7B). To verify these results, we quantified the amount of double strand DNA (dsDNA) present in the samples by PicoGreen ${ }^{\mathrm{TM}}$ assay. This assay allows the quantification of the concentration of dsDNA that can be interpreted as proportional to the number of cells present in the sample. mADSCs from membranes were collected after 7 days of culture and tissue culture plate (TCP) was used as control. The results revealed similar dsDNA concentration of cells growing on ELR5/PAK3 and ELR5/PAK3/GO membranes, suggesting that these membranes facilitate cell adhesion and proliferation (Figure 7A). In contrast, dsDNA concentration of cells growing on both ELR5/PAK2-K4 and ELR5/PAK2-K4/GO membranes was significantly lower than TCP. We hypothesize that the decrease in cellular dsDNA may be the result of (i) a greater cytotoxic effect from the positives charges of PAK4 (Newcomb et al., 2014) or (ii) the higher Young's Modulus of both ELR5/PAK2-K4 and ELR5/PAK2-K4/GO (compared to ELR5/PAK3 and ELR5/PAK3/GO; Figure 6), which could influence cell adhesion. Previous studies have demonstrated that stiffer surfaces can result on lower mADSCs adhesion (Discher et al., 2005).

In summary, these results reveal that incorporation of GO within both ELR5/PAK3 and ELR5/PAK2-K4 systems does not affect the biocompatibility of the material but differences in the resulting architecture and material properties may lead to differences in the capacity of the material to promote cell adhesion and proliferation.

\section{Cell Morphology}

Cells stained with DAPI (nucleus) and Phalloidin CruzFluor ${ }^{\mathrm{TM}}$ 647 (actin) were imaged under an epifluorescent microscope. Analysis of the stained cells revealed that cells grown on ELR5/PAK3 and ELR5/PAK3/GO membranes exhibited a well-spread morphology and formed multiple connections with surrounding cells (Figure 7E). In contrast, cells grown on ELR5/PAK2-K4 and ELR5/PAK2-K4/GO membranes displayed a much less spread morphology and formed fewer connections with neighboring cell. These results suggest that ELR5/PAK3 membranes support better cell adhesion compared to ELR5/PAK2-K4 membranes regardless of the GO content.
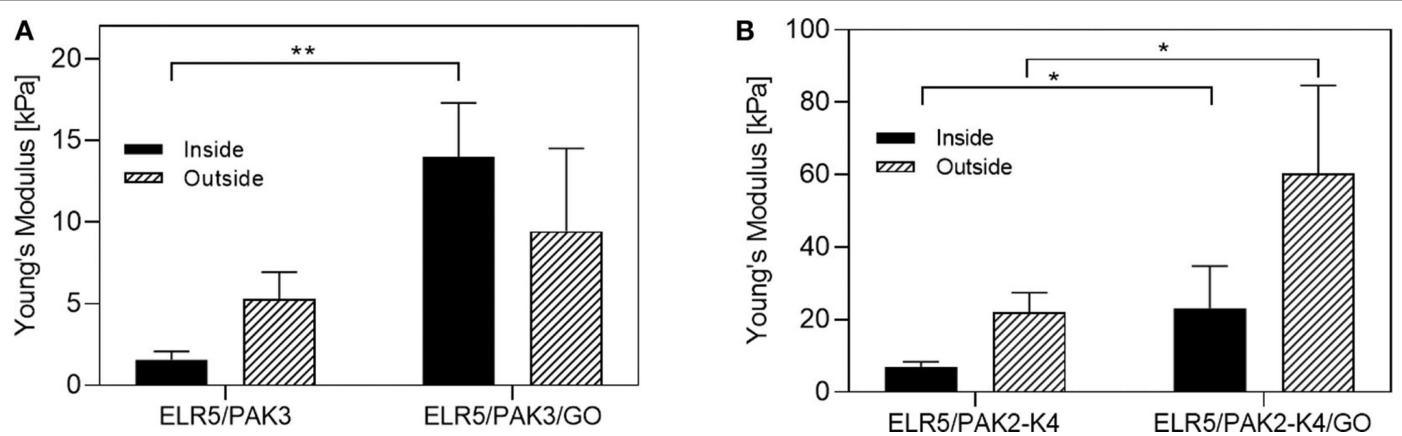

FIGURE 6 | Young's Moduli of the membranes. AFM measurements were carried out on the luminal (inner) and abluminal (outer) side of the ELR5/PAK3 \pm GO membranes (A) and ELR5/PAK2-K4 \pm GO membranes (B). Error bars represent \pm SEM where ${ }^{* *} p<0.01$, and ${ }^{*} p<0.5$. The experiments were performed in 5 replicates. 

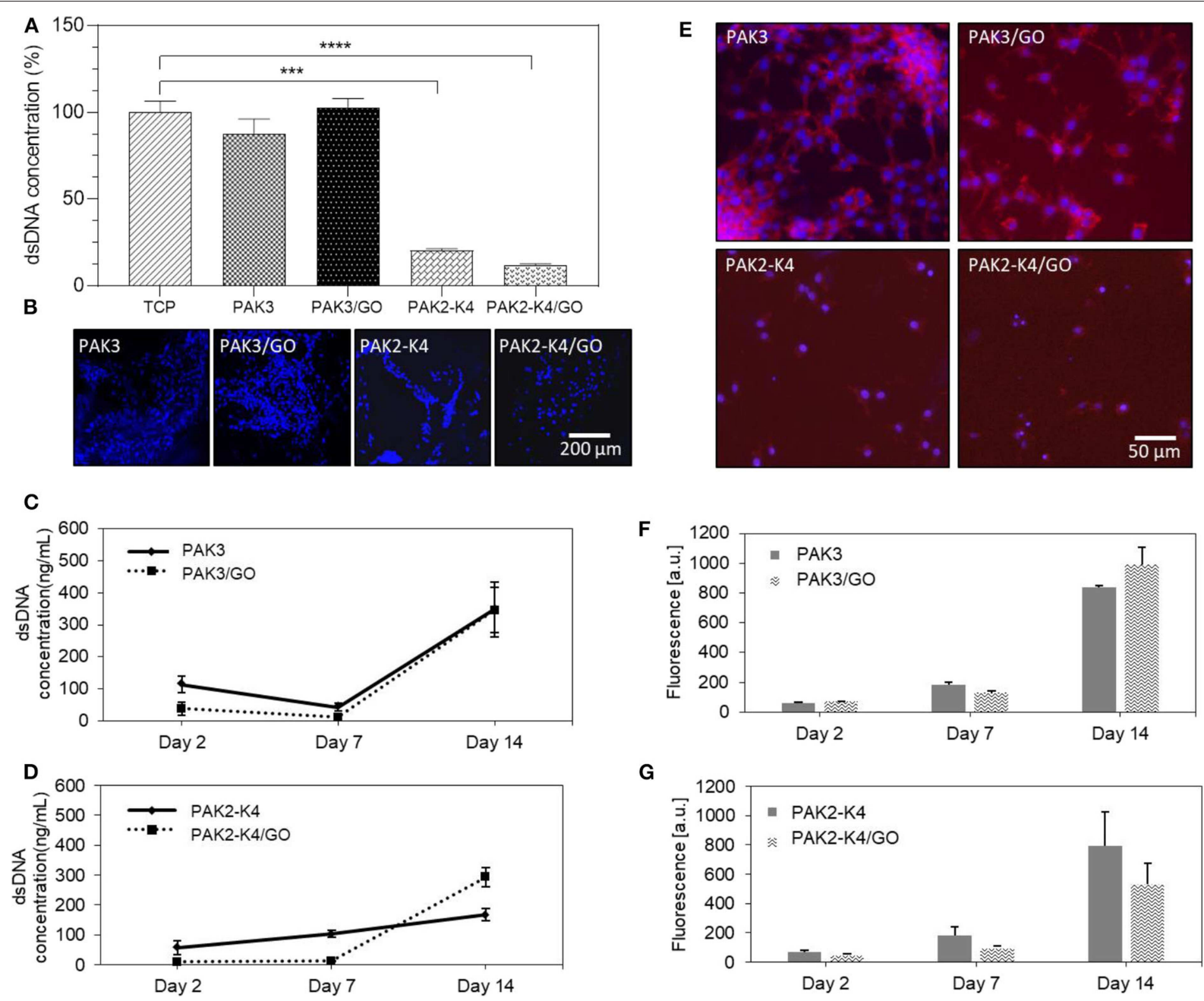

G

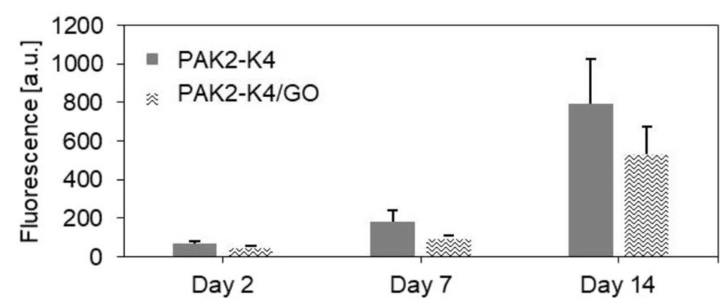

FIGURE 7 | Investigation of biocompatibility of ELR5/PAs/GO membranes. Mouse derived adipose stem cells (mADSCs) were grown on the membranes and dsDNA content (A) was quantified after 7 days in culture and normalized to TCP (100\%) by PicoGreen ${ }^{\text {TM }}$ assay. (B) Fluorescence microscopy images of mADSCs stained with DAPI (4,6-diamidino-2-phenylindole) after $4 \mathrm{~h}$ of cell culture. (C,D) Proliferation studies. mADSCs were grown on the ELR5/PAK3 \pm GO (C) or ELR5/PAK2-K4 \pm GO (D) membranes. dsDNA content was quantified by PicoGreen ${ }^{\text {TM }}$ assay. (E) Morphology studies. mADSCs were stained with Phalloidin CruzFluor ${ }^{\text {TM }} 647$ and DAPI. Images indicate a spreading morphology and intercellular connections between mADSCs growing on ELR5/PAK3 and ELR5/PAK3/GO membranes and a barely visible cytoskeleton of mADSCs grown on ELR5/PAK2-K4 and ELR5/PAK2-K4/GO membranes with minimal spreading and connections observed. (F,G) Cell metabolic activity studies. mADSCs were grown on the ELR5/PAK3 \pm GO (F) or ELR5/PAK2-K4 \pm GO (G) membranes, cell metabolic activity was assessed with Alamar Blue ${ }^{\text {TM }}$ assay. The data suggest there is no significant difference in cell metabolic activity between either ELR5/PAK3 and ELR5/PAK3/GO nor ELR5/PAK2-K4 and ELR5/PAK2-K4/GO systems at any of the time points. Error bars represent \pm SEM where ${ }^{* * *} p<0.0001$ and ${ }^{* * *} p<0.001$. The experiments were performed in triplicates.

\section{Cell Metabolic Activity}

To further assess the capacity of the materials to support cell growth, the metabolic activity of mADSCs on the membranes was assessed by Alamar blue ${ }^{\mathrm{TM}}$ assay over 2 weeks of cell culture. Same membranes used for cell adhesion studies (Figure 7E) revealed no significant difference in metabolic activity for cells growing on the ELR5/PAK3/GO membranes vs. ELR5/PAK3 membranes at any time point (Figure 7F). On the other hand, cells grown on ELR5/PAK2-K4/GO membranes exhibited a slight decrease, though not statistically significant, in metabolic activity compared to those grown on ELR5/PAK2-K4 membranes (Figure 7G). These results further evidence that addition of GO flakes into the ELR5/PAK3 system does not have a negative impact on the metabolic activity of the cells. However, there is a slight decrease in cell metabolic activity after addition of GO in the ELR5/PAK2-K4 system, suggesting supramolecular organization of GO within the membranes or the possible cytotoxicity of the positively charged PAK4 (Newcomb et al., 2014) might play a role.

\section{Cell Proliferation}

Cell proliferation was then assessed by quantifying dsDNA concentration of mADSCs grown on the different membranes on days 2, 7, and 14 via Quant-iT ${ }^{\mathrm{TM}}$ PicoGreen ${ }^{\mathrm{TM}}$ assay. The 
results revealed that in case of the ELR5/PAK3 system, GO does not have an effect on the proliferation of mADSCs at none of the investigated time points (Figure 7C). On the other hand, we observed that GO may be influencing proliferation of mADSCs grown on ELR5/PAK2-K4 membranes, given a decrease in dsDNA concentration at day 7 and an increase at day 14 compared to membranes without GO (Figure 7D). These results indicate that both ELR5/PAK3 and ELR5/PAK2K4 systems support cell proliferation regardless of GO content, although, proliferation rate is slower for cells growing on the ELR5/PAK2-K4 system.

\section{CONCLUSION}

In this study, we report the ability of the ELR5/PA selfassembling system to manipulate, localize, and organize GO flakes into hierarchical structures. By taking advantage of the electrostatic and hydrophobic nature of ELR5 and PAs, the formation of a diffusion barrier upon their co-assembly, and the subsequent diffusion-reaction mechanism of ELR5/PA membrane formation, we demonstrate the potential to use supramolecular mechanisms to guide assembly of GO across scales and into complex architectures. Furthermore, we show that incorporation of GO flakes within the ELR5/PA system improves mechanical properties of the resulting materials and may be beneficial for tissue engineering applications. Also, we demonstrate that incorporation of GO does not affect the capacity of the composite material to support mADSCs adhesion, proliferation, and metabolic activity.

\section{METHODS}

\section{Membrane Formation}

ELR5 and PA molecules were dissolved separately in MilliQ water (10 and $15 \mathrm{mg} / \mathrm{mL}$, respectively). $\mathrm{pH}$ was adjusted to $\mathrm{pH}=$ 5 (ELR5) and $\mathrm{pH}=4.5$ (PAs). $190 \mu \mathrm{l}$ of ELR5 solution was placed in a well in a 48 well plate. $10 \mu \mathrm{l}$ of PA solution was added by immersing the pipette tip into the ELR5 solution and slowly releasing the liquid. The membrane was left to develop for $48 \mathrm{~h}$ at $30^{\circ} \mathrm{C}$. For GO membranes: GO $4 \mathrm{mg} / \mathrm{mL}(\mathrm{pH}=2)$ was diluted to the required concentration with $\mathrm{pH}$ adjusted to $\mathrm{pH}=$ 4.5 and mixed with either ELR5 solution or PA solution before membrane formation. Ten microliter of PA-GO solution was added by immersing the pipette tip into the ELR5-GO solution and slowly releasing the liquid. The membrane was left to develop for $48 \mathrm{~h}$ at RT.

\section{Growth Experiment}

ELR5 and PA molecules were dissolved separately in MilliQ water (10 and $15, \mathrm{mg} / \mathrm{mL}$ respectively). GO $4 \mathrm{mg} / \mathrm{mL}$ was diluted to the required concentration and mixed with either ELR5 solution or PA solution before membrane formation. $100 \mu \mathrm{l}$ of ELR5/GO solution was placed in a glass vial. $5 \mu \mathrm{l}$ of PA-GO solution was added by immersing the pipette tip into the ELR5 solution and slowly releasing the liquid. Additional $20 \mu \mathrm{l}$ of ELR5/GO solution was carefully added to the vial every $10 \mathrm{~min}$ to observe growth of the membrane.

\section{Zeta Potential and Dynamic Light Scattering}

Zeta potential was measured to investigate the changes in surface charge density of ELR5, PAs and GO molecules when mixed together. ELR5 and PAs were dissolved in MilliQ water [0.1 and $0.15 \%(\mathrm{w} / \mathrm{v})$, respectively]. GO was diluted to the final concentration of $0.001 \%(\mathrm{w} / \mathrm{v}) \cdot \mathrm{pH}$ was adjusted to $\mathrm{pH}=5$ (ELR5), $\mathrm{pH}=4.5$ (PAs), and $\mathrm{pH}=4.5$ (GO). All samples were sonicated for $30 \mathrm{~min}$ prior taking the measurement. Zeta potential was measured at $25^{\circ} \mathrm{C}$ on a Zetasizer (Nano-ZS ZEN 3600, Malvern Instruments, UK).

\section{Circular Dichroism Spectroscopy}

ELR5 and PAs were dissolved in MilliQ water [0.025 and 0.01\% $(\mathrm{w} / \mathrm{v})$, respectively]. In order to carry out the measurement at the same conditions as membrane formation, $\mathrm{pH}$ was adjusted to $\mathrm{pH}=5$ (ELR5) and $\mathrm{pH}=4.5$ (PAs). GO was diluted to the final concentration of $0.001 \%(\mathrm{w} / \mathrm{v})$ and $\mathrm{pH}$ was adjusted to $\mathrm{pH}=4.5$. $\mathrm{CD}$ spectra were obtained using $1 \mathrm{~mm}$ path length and $300 \mu \mathrm{l}$ volume quartz cuvette (Chirascan, Applied Photophysics, UK) between 195 and $270 \mathrm{~nm}$ with a $0.5 \mathrm{~nm}$ interval at $25^{\circ} \mathrm{C}$. CD measurement was conducted using Chirascan $^{\mathrm{TM}} \mathrm{CD}$ spectrometer (Chirascan, Applied Photophysics Ltd, UK) equipped with a Peltier temperature controller, under a constant nitrogen purging at a constant pressure of $0.7 \mathrm{MPa}$. Each represented spectrum is the average of three consecutive spectra.

\section{Turbidity}

ELR5 and PA were dissolved in MilliQ water [0.025 and $0.01 \%$ $(\mathrm{w} / \mathrm{v})$, respectively]. GO was diluted to the final concentration of $0.001 \%(\mathrm{w} / \mathrm{v})$. Absorbance of the solutions was measured at $300 \mathrm{~nm}$ using a microplate reader (Spetrostarnano, BMG Labtech, UK) at 4 and $40^{\circ} \mathrm{C}$.

\section{Transmission Electron Microscopy}

Membranes were formed as described. After washing with MilliQ water membranes were crosslinked with TEM grade glutaraldehyde followed by dehydration with a gradient $30-100 \%$ of ethanol. Membranes were then embedded on LRWhite resin and ethanol $50-50 \%$ for $1 \mathrm{~h}$ and $100 \%$ LRW $1 \mathrm{~h}$ and again $100 \%$ LRW overnight. The following day they were encased in capsules filled with resin and left in the oven at $60^{\circ} \mathrm{C}$ for $5 \mathrm{~h}$ to harden. The block was sectioned with a Reicht microtome to a thickness of $70 \mathrm{~nm}$. The sections were loaded onto a copper grid and stained with $2 \%$ uranyl acetate for $4 \mathrm{~min}$ and rinsed in MilliQ water. The samples were visualized on a JEOL JEM 1230 electron microscope operating at $80 \mathrm{kV}$.

\section{Scanning Electron Microscopy}

ELR5/PA/GO membranes were left to develop for $48 \mathrm{~h}$ and then washed in MilliQ water and fixed with $2.5 \%$ glutaraldehyde in MilliQ water for $2 \mathrm{~h}$ at room temperature. Then, the samples were washed in MilliQ water followed by dehydration by immersion in an increasing concentration of ethanol $(20,50,70,90,96$, and $100 \%)$. The samples were then subjected to a process of critical point drying (K850, Quorum Technologies, UK) followed by 
sputter-coating with gold for $90 \mathrm{~s}$. SEM imaging was carried out using an Inspect F50 (FEI Comp, The Netherlands).

\section{GO Distribution}

Membranes were formed as previously described. After washing in MilliQ water membranes were cut open, put flat on a microscope slide and then covered with a slide cover. 3D images were obtained with Zeiss LSM710 confocal microscope.

\section{GO Mass}

Fully formed and washed membranes were dissolved in 1,1,1,3,3,3-hexafluoro-2-propanol (HFIP) followed by sonication for $30 \mathrm{~min}$. GO absorbance was measured spectrophotometrically in the UV-Vis region. Standard curve was prepared using a solution of GO flakes in HFIP at a known concentration.

\section{Atomic Force Microscopy}

Atomic force microscopy was used to measure Young's Modulus of the investigated system. Membranes were attached to a Petri dish using a drop of cyanoacrylate adhesive and left for a minute for the adhesive to dry followed by immersion in ultrapure MilliQ water. Young's Modulus measurements were taken with JPK Nanowizard-1 (JPK Instruments, Germany) in force spectroscopy mode, which was mounted on an inverted optical microscope (IX-81, Olympus, Japan). Indentation was carried out using quadratic pyramidal cantilevers (MLCT, Bruker, MA, USA) with spring constant of $0.07 \mathrm{~N} / \mathrm{m}$ and half-angle to face of $17.5^{\circ}$. Measurements were taken in multiple areas per sample and multiple times per area.

\section{Cell Studies}

Fully developed membranes were washed with MilliQ water and crosslinked with genipin at a concentration of $25 \mu \mathrm{l} / \mathrm{ml}$ at $37^{\circ} \mathrm{C}$ overnight. Tubes were then washed in MilliQ water and sterilized under UV light for $20 \mathrm{~min}$. After sterilization, tubes were washed three times in Hank's balanced salt solution. 50,000 mADSCs resuspended in DMEM (10\% FBS, 1\% P/S) were seeded on each ELR5/PA/GO tube. Media was changed every 2 to 3 days.

\section{Cell Attachment}

Cells were seeded as previously described in a serum free DMEM media and incubated for $4 \mathrm{~h}$ followed by additional $4 \mathrm{~h}$ in full media (DMEM with 20\% FBS). Cells were fixed with $4 \%$ paraform aldehyde for $1 \mathrm{~h}$ and stained with blue dye 4'-6diamino-2-phenylindole (DAPI), followed by imaging under an epifluorescent microscope (Leica DMi8).

Cell morphology was assessed by using epifluorescent microscopy (Leica DMi8). After the cell culture membranes were fixed with $4 \%$ paraform aldehyde for $1 \mathrm{~h}$ and stained with DAPI and Phalloidin CruzFluor ${ }^{\mathrm{TM}} 647$.

\section{REFERENCES}

Azarniya, A., Eslahi, N., Mahmoudi, N., and Simchi, A. (2016). Effect of graphene oxide nanosheets on the physico-mechanical properties of chitosan/bacterial
Cell metabolic activity was assessed on days 2, 7, and 14 with AlamarBlue $^{\mathrm{TM}}$ cell metabolic assay. Membranes were incubated for $2 \mathrm{~h}$ at $37^{\circ} \mathrm{C}$ in a $10 \%(\mathrm{v} / \mathrm{v})$ solution of AlamarBlue ${ }^{\mathrm{TM}}$ in DMEM. Fluorescence of the solution was then read at 570 and $595 \mathrm{~nm}$ using a microplate reader (Spetrostarnano, BMG Labtech, UK).

Cell proliferation was assessed by quantifying the number of adherent cells to membranes with Quant-iT ${ }^{\mathrm{TM}}$ PicoGreen ${ }^{\mathrm{TM}}$ assay on days 2, 7, and 14. Briefly, cells were lysed and the supernatant solution was diluted in assay buffer followed by addition of Quant-iT ${ }^{\mathrm{TM}}$ PicoGreen ${ }^{\mathrm{TM}}$ reagent and incubation for $5 \mathrm{~min}$ at RT. Fluorescence of the samples was measured at $480 \mathrm{~nm}$ (excitation) and $520 \mathrm{~nm}$ (emission) using a microplate reader (Spetrostarnano, BMG Labtech, UK). The DNA concentration for each sample was calculated by using a standard curve.

\section{DATA AVAILABILITY STATEMENT}

The raw data supporting the conclusions of this article will be made available by the authors, without undue reservation.

\section{AUTHOR CONTRIBUTIONS}

AMat, ES, AD, and AMaj designed the project and wrote the article. CR-G synthesized Pas. JR-C provided ELR. EC and KI-B helped with data interpretation. AMaj, MG, and AR conducted experiments and analyzed data.

\section{ACKNOWLEDGMENTS}

This work was supported by the ERC Starting Grant (STROFUNSCAFF), the Marie Curie Career Integration Grant (BIOMORPH), the AO Research Fund of the AO Foundation project number AOCMF-17-19M, and the UK Regenerative Medicine Platform (UKRMP2) Acellular Smart Materials. Also, the authors are grateful for funding from the Spanish Government (MAT2016-78903-R), Junta de Castilla y León (VA317P18), Interreg V A España Portugal POCTEP (0624_2IQBIONEURO_6_E), and Centro en Red de Medicina Regenerativa y Terapia Celular de Castilla y León. Furthermore, we would like to acknowledge the Program for Innovation and Human Capital from the Ministry of Science, Technology, and Telecommunications of the Republic of Costa Rica (MICITT-PINN-PED-014-2015-2).

\section{SUPPLEMENTARY MATERIAL}

The Supplementary Material for this article can be found online at: https://www.frontiersin.org/articles/10.3389/fmats. 2020.00167/full\#supplementary-material

cellulose nanofibrous composites. Compos. Part A Appl. Sci. Manuf. 85, 113-122. doi: 10.1016/j.compositesa.2016.03.011

Azevedo, H. S. (2019). "Biomaterials inspired by biology: from molecules to self-assembly," Reference Module in Biomedical Sciences (Elsevier), 109-117. 
Capito, R. M., Azevedo, H. S., Velichko, Y. S., Mata, A., and Stupp, S. I. (2008). Self-assembly of large and small molecules into hierarchically ordered sacs and membranes. Science 319, 1812-1816. doi: 10.1126/science.11 54586

Depan, D., Pesacreta, T. C., and Misra, R. D. K. (2014). The synergistic effect of a hybrid graphene oxide-chitosan system and biomimetic mineralization on osteoblast functions. Biomater. Sci. 2, 264-274. doi: 10.1039/C3BM60192G

Dinescu, S., Ionita, M., Pandele, A. M., Galateanu, B., Iovu, H., Ardelean, A., et al. (2014). In vitro cytocompatibility evaluation of chitosan/graphene oxide 3D scaffold composites designed for bone tissue engineering. Biomed. Mater. Eng. 24, 2249-2256. doi: 10.3233/BME-141037

Discher, D. E., Janmey, P., and Wang, Y. L. (2005). Tissue cells feel and respond to the stiffness of their substrate. Science 310, 1139-1143. doi: 10.1126/science.1116995

Draper, E. R., Eden, E. G., McDonald, T. O., and Adams, D. J. (2015). Spatially resolved multicomponent gels. Nat. Chem. 7, 848-852. doi: $10.1038 /$ nchem. 2347

Gazit, E. (2007). Self-assembled peptide nanostructures: the design of molecular building blocks and their technological utilization. Chem. Soc. Rev. 36:1263. doi: 10.1039/b605536m

Gentile, P., Ferreira, A. M., Callaghan, J. T., Miller, C. A., Atkinson, J., Freeman, C., et al. (2017). Multilayer nanoscale encapsulation of biofunctional peptides to enhance bone tissue regeneration in vivo. Adv. Healthc. Mater. 6:1601182. doi: 10.1002/adhm.201601182

Han, D., Yan, L., Chen, W., and Li, W. (2011). Preparation of chitosan/graphene oxide composite film with enhanced mechanical strength in the wet state. Carbohydr. Polym. 83, 653-658. doi: 10.1016/j.carbpol.2010.08.038

Hedegaard, C. L., Collin, E. C., Redondo-Gómez, C., Nguyen, L. T., Ng, K. W., Castrejón-Pita, A. A., et al. (2018). Hydrodynamically guided hierarchical self-assembly of peptide-protein bioinks. Adv. Funct. Mater. 28:1703716. doi: 10.1002/adfm.201703716

Inostroza-Brito, K. E., Collin, E., Siton-Mendelson, O., Smith, K. H., MongeMarcet, A., Ferreira, D. S., et al. (2015). Co-Assembly, spatiotemporal control and morphogenesis of a hybrid protein-peptide system. Nat. Chem. 7, 897-904. doi: 10.1038/nchem. 2349

Inostroza-Brito, K. E., Collin, E. C., Majkowska, A., Elsharkawy, S., Rice, A., Armando, E., et al. (2017). Cross-linking of a biopolymer-peptide coassembling system. Acta Biomater. 58, 80-89. doi: 10.1016/j.actbio.2017.05.043

Jalaja, K., Sreehari, V. S., Kumar, P. R. A., and Nirmala, R. J. (2016). Graphene oxide decorated electrospun gelatin nanofibers: fabrication, properties and applications. Mater. Sci. Eng. C 64, 11-19. doi: 10.1016/j.msec.2016.03.036

Justino, C. I. L., Gomes, A. R., Freitas, A. C., Duarte, A. C., and Rocha-Santos, T. A. P. (2017). Graphene based sensors and biosensors. TrAC Trends Anal. Chem. 91, 53-66. doi: 10.1016/j.trac.2017.04.003

Kang, S., Park, J. B., Lee, T. J., Ryu, S., Bhang, S. H., La, W. G., et al. (2015). Covalent conjugation of mechanically stiff graphene oxide flakes to three-dimensional collagen scaffolds for osteogenic differentiation of human mesenchymal stem cells. Carbon 83, 162-172. doi: 10.1016/j.carbon.2014.11.029

Kumar, M., Ing, N. L., Narang, V., Wijerathne, N. K., Hochbaum, A. I., and Ulijn, R. V. (2018). Amino-acid-encoded biocatalytic self-assembly enables the formation of transient conducting nanostructures. Nat. Chem. 10, 696-703. doi: 10.1038/s41557-018-0047-2

Lee, J. H., Lee, Y., Shin, Y. C., Kim, M. J., Park, J. H., Hong, S. W., et al. (2016). In situ forming gelatin/graphene oxide hydrogels for facilitated C2C12 myoblast differentiation. Appl. Spectrosc. Rev. 51, 527-539. doi: 10.1080/05704928.2016.1165686

Li, J., Ren, N., Qiu, J., Mou, X., and Liu, H. (2013). Graphene oxide-reinforced biodegradable genipin-cross-linked chitosan fluorescent biocomposite film and its cytocompatibility. Int. J. Nanomed. 8, 3415-3426. doi: 10.2147/IJN.S51203

Lin, J., Chen, X., and Huang, P. (2016). Graphene-based nanomaterials for bioimaging. Adv. Drug Deliv. Rev. 105, 242-254. doi: 10.1016/j.addr.2016.05.013

Liu, J., Cui, L., and Losic, D. (2013). Graphene and graphene oxide as new nanocarriers for drug delivery applications. Acta Biomater. 9, 9243-9257. doi: 10.1016/j.actbio.2013.08.016

Mata, A., Hsu, L., Capito, R., Aparicio, C., Henrikson, K., and Stupp, S. I. (2009). Micropatterning of bioactive self-assembling gels. Soft Matter 5, 1228-1236. doi: $10.1039 / \mathrm{b} 819002 \mathrm{j}$
Mata, A., Palmer, L., Tejeda-Montes, E., and Stupp, S. I. (2012). Design of biomolecules for nanoengineered biomaterials for regenerative medicine. Methods Mol. Biol. 811, 39-49. doi: 10.1007/978-1-61779-388-2_3

Mazaheri, M., Akhavan, O., and Simchi, A. (2014). Flexible bactericidal graphene oxide-chitosan layers for stem cell proliferation. Appl. Surf. Sci. 301, 456-462. doi: 10.1016/j.apsusc.2014.02.099

Mendes, A. C., Baran, E. T., Reis, R. L., and Azevedo, H. S. (2013). Selfassembly in nature: using the principles of nature to create complex nanobiomaterials. Wiley Interdiscip. Rev. Nanomed. Nanobiotechnol. 5, 582-612. doi: 10.1002/wnan.1238

Navon, Y., and Bitton, R. (2016). Elastin-like peptides (ELPs) building blocks for stimuli-responsive self-assembled materials. Isr. J. Chem. 56, 581-589. doi: 10.1002/ijch.201500016

Newcomb, C. J., Sur, S., Ortony, J. H., Lee, O. S., Matson, J. B., Boekhoven, J., et al. (2014). Cell death versus cell survival instructed by supramolecular cohesion of nanostructures. Nat. Commun. 5:3321. doi: 10.1038/ncomms4321

Okesola, B. O., Lau, H. K., Derkus, B., Boccorh, D. K., Wu, Y., Wark, A. W., et al. (2020a). Covalent co-assembly between resilin-like polypeptide and peptide amphiphile into hydrogels with controlled nanostructure and improved mechanical properties. Biomater. Sci. 8, 846-857. doi: 10.1039/C9BM01796H

Okesola, B. O., and Mata, A. (2018). Multicomponent self-assembly as a tool to harness new properties from peptides and proteins in material design. Chem. Soc. Rev. 47, 3721-3736. doi: 10.1039/C8CS00121A

Okesola, B. O., Ni, S., Derkus, B., Galeano, C. C., Hasan, A., Wu, Y., et al. (2020b). Growth-factor free multicomponent nanocomposite hydrogels that stimulate bone formation. Adv. Funct. Mater. 30:1906205. doi: 10.1002/adfm.201906205

Okesola, B. O., Wu, Y., Derkus, B., Gani, S., Wu, D., Knani, D., et al. (2019). Supramolecular self-assembly to control structural and biological properties of multicomponent hydrogels. Chem. Mater. 31, 7883-7897. doi: 10.1021/acs.chemmater.9b01882

Pashuck, E. T., Cui, H., and Stupp, S. I. (2010). Tuning supramolecular rigidity of peptide fibers through molecular structure. J. Am. Chem. Soc. 132, 6041-6046. doi: $10.1021 /$ ja908560n

Perczel, A., and Fasman, G. D. (1992). Quantitative analysis of cyclic beta-turn models. Protein Sci. 26, 1527-1572.

Rajan Unnithan, A., Ramachandra Kurup Sasikala, A., Park, C. H., and Kim, C. S. (2017). A unique scaffold for bone tissue engineering: an osteogenic combination of graphene oxide-hyaluronic acid-chitosan with simvastatin. J. Ind. Eng. Chem. 46, 182-191. doi: 10.1016/j.jiec.2016.10.029

Raymond, D. M., and Nilsson, B. L. (2018). Multicomponent peptide assemblies. Chem. Soc. Rev. 47, 3659-3720. doi: 10.1039/C8CS00115D

Redondo-Gómez, C., Abdouni, Y., Becer, C. R., and Mata, A. (2019). Self-assembling hydrogels based on a complementary hostguest peptide amphiphile pair. Biomacromolecules 20, 2276-2285. doi: 10.1021/acs.biomac.9b00224

Schneider, C. A., Rasband, W. S., and Eliceiri, K. W. (2012). NIH image to imageJ: 25 years of image analysis. Nat. Methods 9, 671-675. doi: 10.1038/nmeth.2089

Shao, L., Chang, X., Zhang, Y., Huang, Y., Yao, Y., and Guo, Z. (2013). Graphene oxide cross-linked chitosan nanocomposite membrane. Appl. Surf. Sci. 280, 989-992. doi: 10.1016/j.apsusc.2013.04.112

Shin, S. R., Li, Y. C., Jang, H. L., Khoshakhlagh, P., Akbari, M., Nasajpour, A., et al. (2016). Graphene-based materials for tissue engineering. Adv. Drug Deliv. Rev. 105, 255-274. doi: 10.1016/j.addr.2016.03.007

Stankovich, S., Piner, R. D., Nguyen, S. B. T., and Ruoff, R. S. (2006). Synthesis and exfoliation of isocyanate-treated graphene oxide nanoplatelets. Carbon 44, 3342-3347. doi: 10.1016/j.carbon.2006.06.004

Stephanopoulos, N., Ortony, J. H., and Stupp, S. I. (2013). Self-assembly for the synthesis of functional biomaterials. Acta Mater. 61, 912-930. doi: 10.1016/j.actamat.2012.10.046

Tang, H., Liu, D., Zhao, Y., Yang, X., Lu, J., and Cui, F. (2015). Molecular dynamics study of the aggregation process of graphene oxide in water. J. Phys. Chem. C 119, 26712-26718. doi: 10.1021/acs.jpcc.5b07345

Wan, C., Frydrych, M., and Chen, B. (2011). Strong and bioactive gelatin-graphene oxide nanocomposites. Soft Matter 7, 6159-6166. doi: 10.1039/c1sm05321c

Webber, M. J., Tongers, J., Renault, M. A., Roncalli, J. G., Losordo, D. W., and Stupp, S. I. (2010). Development of bioactive peptide amphiphiles for therapeutic cell delivery. Acta Biomater. 6, 3-11. doi: $10.1016 /$ j.actbio.2009.07.031 
Whitesides, G. M., and Grzybowski, B. (2002). Self-assembly at all scales. Science 295, 2418-2421. doi: 10.1126/science.1070821

Wick, P., Louw-Gaume, A. E., Kucki, M., Krug, H. F., Kostarelos, K., Fadeel, B., et al. (2014). Classification framework for graphene-based materials. Angew. Chem. Int. Ed. 53, 7714-7718. doi: 10.1002/anie.201403335

Yang, Y., Asiri, A. M., Tang, Z., Du, D., and Lin, Y. (2013). Graphene based materials for biomedical applications. Mater. Today 16, 365-373. doi: 10.1016/j.mattod.2013.09.004

Zhang, S. (2003). Fabrication of novel biomaterials through molecular selfassembly. Nat. Biotechnol. 21, 1171-1178. doi: 10.1038/nbt874

Zhang, S., Greenfield, M. A., Mata, A., Palmer, L. C., Bitton, R., Mantei, J. R., et al. (2010). A self-assembly pathway to aligned monodomain gels. Nat. Mater. 9, 594-601. doi: 10.1038/nmat2778

Zhou, X., Nowicki, M., Cui, H., Zhu, W., Fang, X., Miao, S., et al. (2017). 3D bioprinted graphene oxide-incorporated matrix for promoting chondrogenic differentiation of human bone marrow mesenchymal stem cells. Carbon 116, 615-624. doi: 10.1016/j.carbon.2017.02.049
Zhu, Y., Murali, S., Cai, W., Li, X., Suk, J. W., Potts, J. R., et al. (2010). Graphene and graphene oxide: synthesis, properties, and applications. Adv. Mater. 22, 3906-3924. doi: 10.1002/adma.201001068

Conflict of Interest: The authors declare that the research was conducted in the absence of any commercial or financial relationships that could be construed as a potential conflict of interest.

Copyright (c) 2020 Majkowska, Redondo-Gómez, Rice, Gonzalez, InostrozaBrito, Collin, Rodriguez-Cabello, Del Rio Hernandez, Solito and Mata. This is an open-access article distributed under the terms of the Creative Commons Attribution License (CC BY). The use, distribution or reproduction in other forums is permitted, provided the original author(s) and the copyright owner(s) are credited and that the original publication in this journal is cited, in accordance with accepted academic practice. No use, distribution or reproduction is permitted which does not comply with these terms. 